\title{
A Case of Marked Hyperlipoprotein(a)emia Associated with Nephrotic Syndrome and Advanced Atherosclerosis
}

\author{
Yoko Kuge, Shuichi Nozaki, Akinori Kitagawa, Takuya Inoue, Hiroyuki Otsuka, and \\ Yoshiharu Ito \\ Department of Internal Medicine, Kawanishi City General Hospital, Hyogo, Japan.
}

\begin{abstract}
In 1989, we encountered a 68-year-old male patient with marked hyperlipoprotein(a)emia (hyperLp(a)emia), who was being treated for hypertension and arteriosclerotic obliterans (ASO) at an outpatient clinic of our hospital. He began to develop leg edema in 2002 and was referred to the Department of Internal Medicine. It was determined that he had severe hyperlipidemia (total cholesterol, $362 \mathrm{mg} / \mathrm{dl}$ ), proteinuria, and hypoalbuminemia, suggesting the presence of nephrotic syndrome. On lipoprotein analysis, he was found to have very high levels of Lp(a) in the plasma (329 mg/dl). Severe atherosclerosis was also found: that is, abdominal aortic aneurysm (AAA) and coronary artery disease (CAD) were detected, in addition to ASO. After remission of the nephrotic syndrome, the plasma Lp(a) level decreased to $204 \mathrm{mg} / \mathrm{dl}$ and the total cholesterol concentration decreased to $179 \mathrm{mg} / \mathrm{dl}$, while very high levels of Lp(a) persisted. We estimate that the markedly elevated Lp(a) plasma levels in this patient may have played some role in the progression of atherosclerosis. $J$ Atheroscler Thromb, 2004; 11: 293-298.
\end{abstract}

Key words: Nephrotic syndrome, Atherosclerosis, Lp(a), Arteriosclerotic obliterans

\section{Introduction}

Lipoprotein (a) $[\mathrm{Lp}(\mathrm{a})]$ is a unique macromolecule formed by a lipoprotein particle with apo(a), apo $B$ and lipids comprising low density lipoprotein (LDL). Apo(a) has a high degree of structural homology with plasminogen, a key enzyme of the coagulation cascade (1). Numerous clinical studies have provided evidence that an elevated level of $L p(a)$ in plasma is an important risk factor for atherosclerosis $(2,3)$. However, there have been few reports describing the clinical picture in patients with extremely high levels of $L p(a)$.

In the present study, we describe a case showing marked hyperlipoprotein(a)emia [hyperLp(a)emia] during the course of nephrotic syndrome. The levels of plasma

Address for correspondence: Shuichi Nozaki, Department of Internal Medicine, Kawanishi City General Hospital, Higashi-uneno 5-21-1, Kawanishi City, Hyogo 666-0195, Japan.

E-mail: shunoza@aa.pial.jp

Received January 22, 2004.

Accepted for publication May 12, 2004. lipoproteins were followed in this patient to elucidate the underlying relationship between severe atherosclerosis and elevated plasma $L p(a)$ levels.

\section{Materials and Methods}

Blood was drawn from the subject after an overnight fast. Lipoprotein fractions were serially isolated by ultracentrifugation. Serum total cholesterol (TC), triglyceride (TG), and high density lipoprotein-cholesterol (HDL-C) were determined by enzymatic methods. Lp(a) was determined by a latex immunoassay method (4). Lp(a) phenotyping was performed by SDS-PAGE with immunoblotting using the method described by Abe et al. (5), which is similar to the procedure by Utermann et al. (6)

\section{Case Presentation}

An 83-year-old male patient had a 22-year history of hypertension. In December 1989, at 68 years of age, the 
patient developed intermittent claudication, and a diagnosis of arteriosclerosis obliterans (ASO) was made by angiography, which showed stenosis of the bilateral external iliac artery. Therefore, he was followed by the Department of Surgery of this hospital. Two coronary risk factors were noted: smoking 10 cigarettes per day for about 40 years, and mild hypertension. His serum TC, TG, and HDL-C levels were 200, 60 and $64 \mathrm{mg} / \mathrm{dl}$, respectively. The LDL-cholesterol level calculated by the Friedewald formula was $124 \mathrm{mg} / \mathrm{dl}$. During the 10-year follow-up, the plasma cholesterol concentration ranged between 200 and 220 mg/dl. Plasma TG and HDL-C concentrations were within normal limits. Blood glucose levels were also within normal limits. The patient stopped smoking after ASO was diagnosed. One year before the admission in September 2001, abdominal aortic aneurysm (AAA) was found by computed tomogaphy (CT). At the age of 81 years, in 2002, he was referred to the Department of Internal Medicine because of leg edema. On examination, he demonstrated hypercholesterolemia (362 $\mathrm{mg} / \mathrm{dl})$, proteinuria (3.5 g/day), and hypoalbuminemia (2.6 $\mathrm{g} / \mathrm{dl}$ ), suggesting the presence of nephrotic syndrome. The level of Lp(a) was $329 \mathrm{mg} / \mathrm{dl}$ and the patient was admitted to hospital. His elder brother, who died of coronary artery disease (CAD), had also been diagnosed with ASO.

This patient's body weight was $50.5 \mathrm{~kg}$ and height was $158 \mathrm{~cm}$. Body temperature was $36.5^{\circ} \mathrm{C}$ and blood pressure was 156/84 mmHg. Edema of the lower left leg was noted and the pedal pulses were weak. There were no skin lesions. Typical fundoscopical findings of pseudoxanthoma elasticum, termed angioid streaks, were not found. Respiratory and heart sounds were normal. Abdominal examination demonstrated increased pulsation around the umbilical area. On neurological examination, there were no abnormalities.

The urine was positive $(+++)$ for protein; the sediment contained no red blood cells, white blood cells or casts. Hematological, blood chemical, and enzyme values and the results of serological testing on admission are given in Fig. 1. The serum protein level was decreased $(5.0 \mathrm{~g} /$ $\mathrm{dl}$ ) and the plasma albumin concentration was $2.6 \mathrm{~g} / \mathrm{dl}$. Hematological indices remained within normal ranges. The treponema pallidum hemagglutination test and serological test for syphilis were negative. Hypercholesterolemia was noted. The blood glucose level was $94 \mathrm{mg} / \mathrm{dl}$ and $\mathrm{Hb}_{\mathrm{A} 1 \mathrm{c}}$ was $5.4 \%$. The plasma IgA level was normal (226 mg/dl). Plasma C3, C4 levels were within normal limits (84 and $29 \mathrm{mg} / \mathrm{dl}$, respectively). Lipoprotein disc electrophoresis showed the presence of midband. On lipoprotein fractionation, very low density lipoprotein (VLDL), intermediate density lipoprotein (IDL), LDL, HDL2 and HDL3 cholesterol concentrations were 17, 10, 211, 66 and $19 \mathrm{mg} / \mathrm{dl}$, respectively. The IDL cholesterol concentration was not increased, suggesting that the midband was due to high levels of $L p(a)$ and not to remnant accumulation. The Lp(a) phenotype was F, S3. Chest $\mathrm{X}$-ray demonstrated calcification in the aortic arch.

Ultrasonographic examination of the carotid artery showed diffuse thickening of the intimal-media complex,

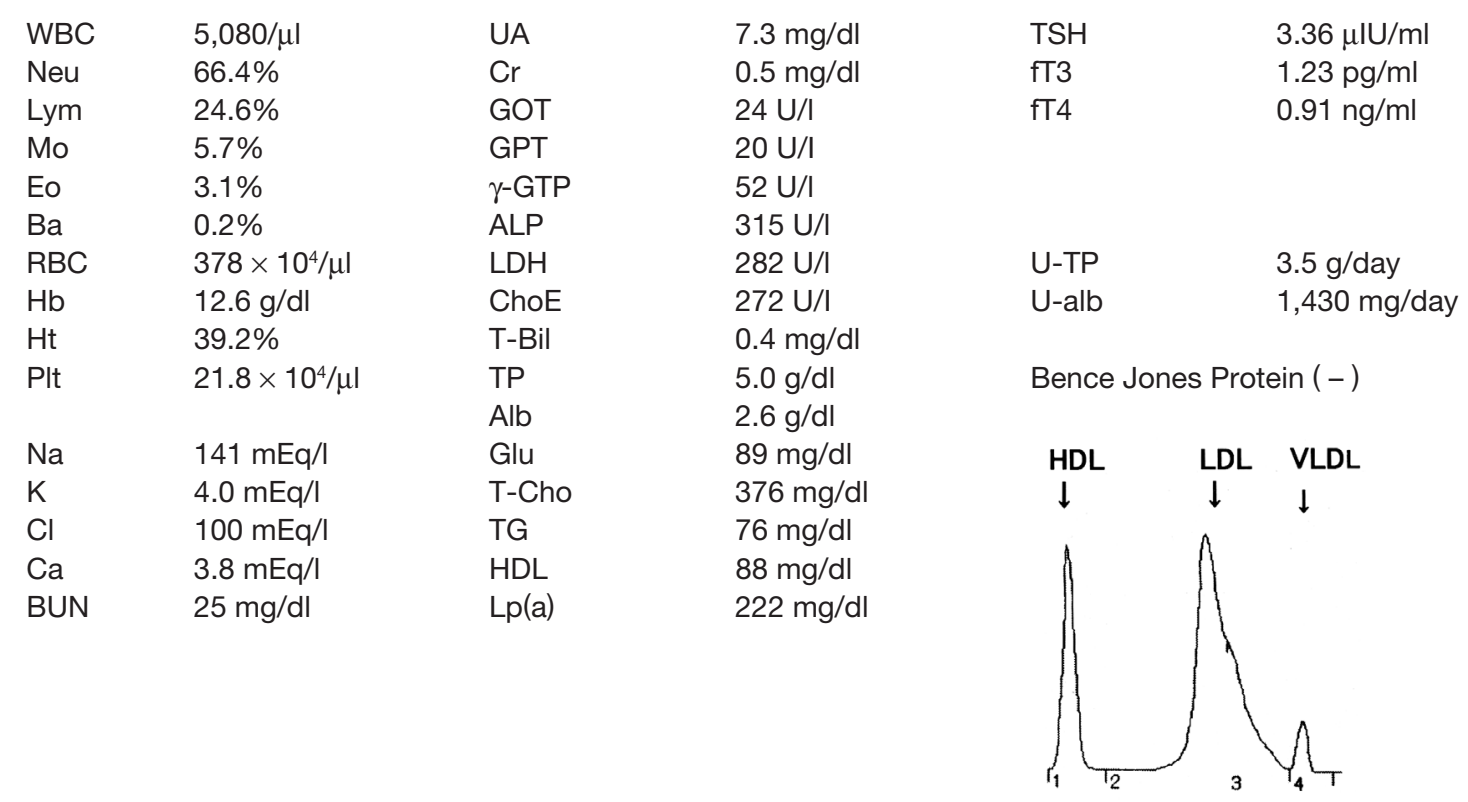

Fig. 1. Laboratory data and lipoprotein disc electrophoresis. U-TP: urinary total protein, U-alb: urinary albumin 
and plaques of the vessel walls in the bilateral carotid arteries (Figs. 2a, 2b). Magnetic resonance angiography (MRA) of the lower limb demonstrated stenosis in the right external iliac artery and occlusion in the left external iliac artery (Fig. 3). Abdominal CT demonstrated AAA with a diameter of about $4 \mathrm{~cm}$ (Fig. 4). Chest CT showed remarkable calcification of the right and left coronary arteries (Figs. 5a, 5b). Radionuclide imaging with ${ }^{123} \mathrm{l}$-labeled beta methyl-iodophenyl penta-decanoic acid (BMIPP), and thallium(TI)-201 single-photon emission computed tomography (SPECT) showed less uptake of BMIPP than of TI-201 in the inferior area, indicating myocardial ischemia. Since the patient did not present any symptoms of angina pectoris and he declined more detailed examination, coronary arteriography was not performed.

Figure 6 shows the clinical course. Dipiridamole (300 $\mathrm{mg} /$ day) and imidapril (5 mg/day) were prescribed, and a protein-restricted diet (less than $20 \mathrm{~g} /$ day of protein) was

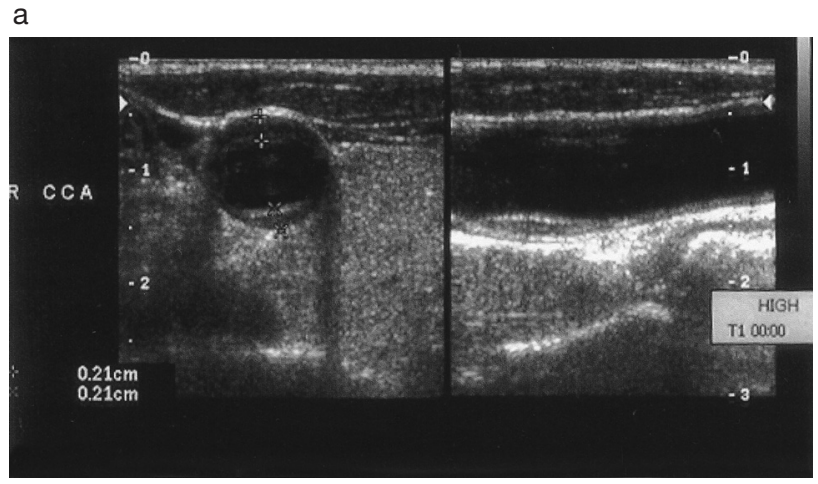

b

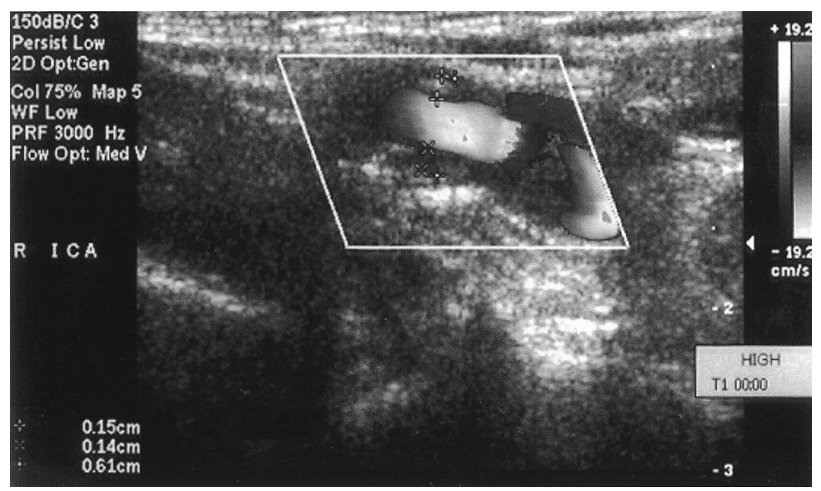

Fig. 2. Ultrasonographic examination of the carotid artery showed diffuse thickening of the intimal-media complex, and plaques in the right common carotid artery (a) and right internal carotid artery (b).

There were also plaques and thickening of intimal-media complex in the left carotid artery (data not shown). provided. The proteinuria gradually decreased and the levels of $L p(a)$ decreased. The leg edema improved and he was discharged in May 2002. After two months, plasma albumin levels decreased again and the leg edema became prominent. Therefore, he was readmitted in August 2002. On this second admission, the highest level of $L p(a)$ was $356 \mathrm{mg} / \mathrm{dl}$. Again, the albumin levels were decreased and cholesterol levels were increased. Almost the same regimen as that in the first administration was prescribed, and the proteinuria improved, while TC and $L p(a)$ levels decreased.

Ten months after discharge, the nephrotic syndrome was almost in complete remission: albumin levels were normalized and cholesterol levels had decreased to almost normal (179 mg/dl). The Lp(a) levels decreased with the increase in plasma albumin levels. However, the $L p(a)$ levels remained markedly elevated $(204 \mathrm{mg} / \mathrm{dl})$.

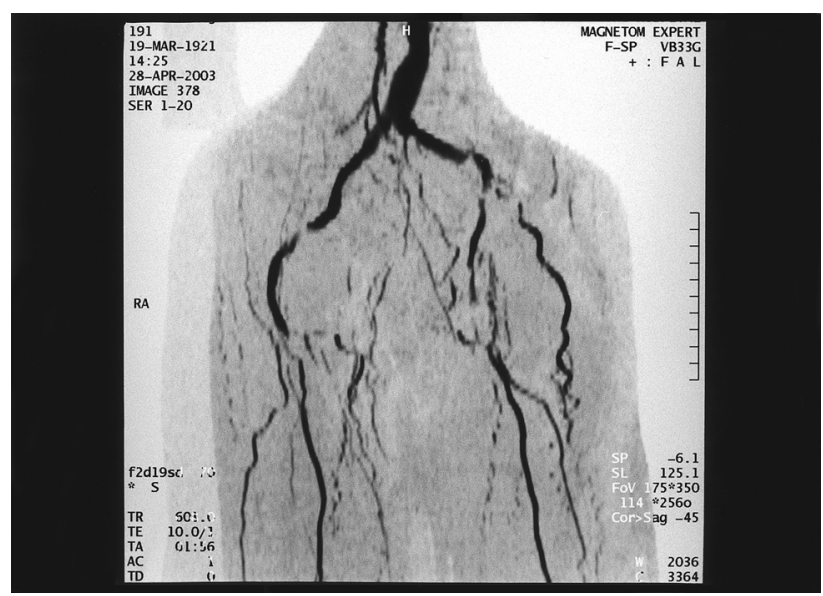

Fig. 3. MRA of the lower limb demonstrated stenosis in the right external iliac artery and occlusion in the left external iliac artery.

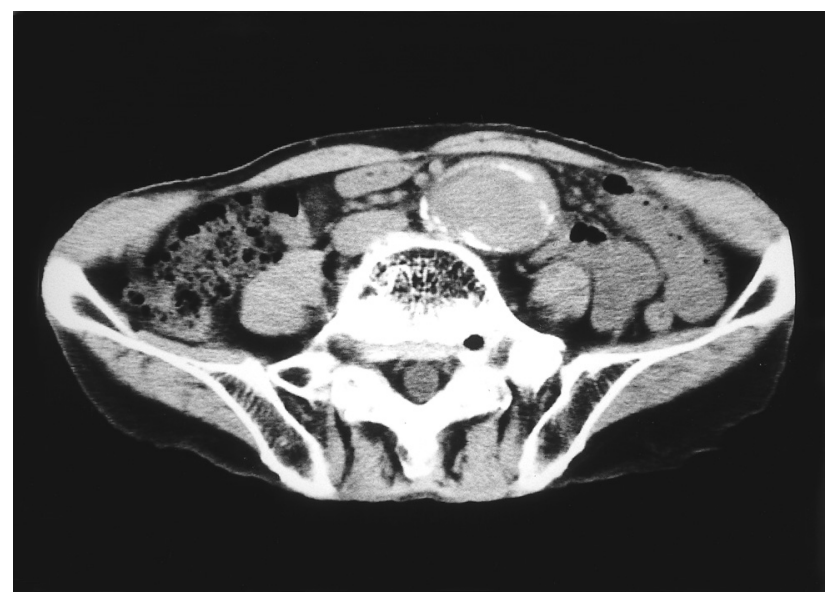

Fig. 4. Abdominal CT showing AAA with a diameter of about 4 $\mathrm{cm}$. 
a

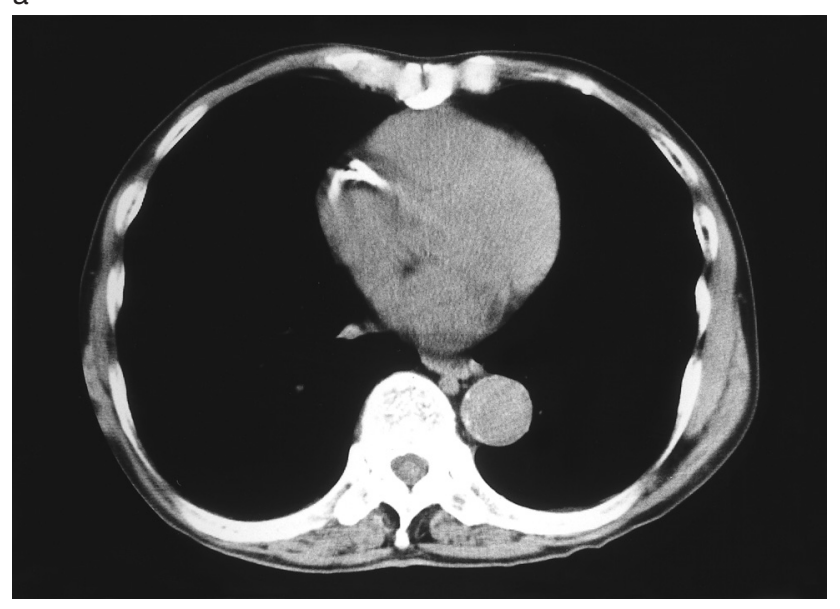

b

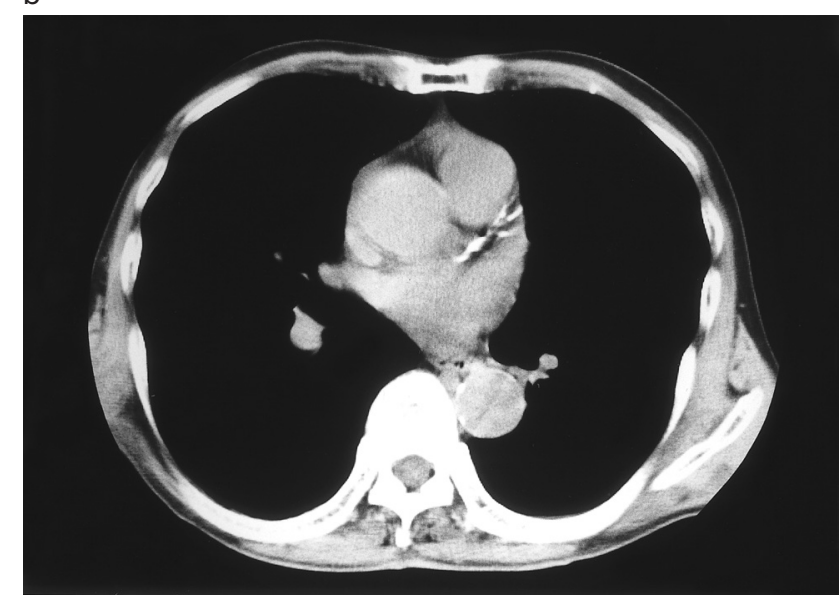

Fig. 5. Chest CT showed severe calcification of the right (a) and left (b) coronary arteries.

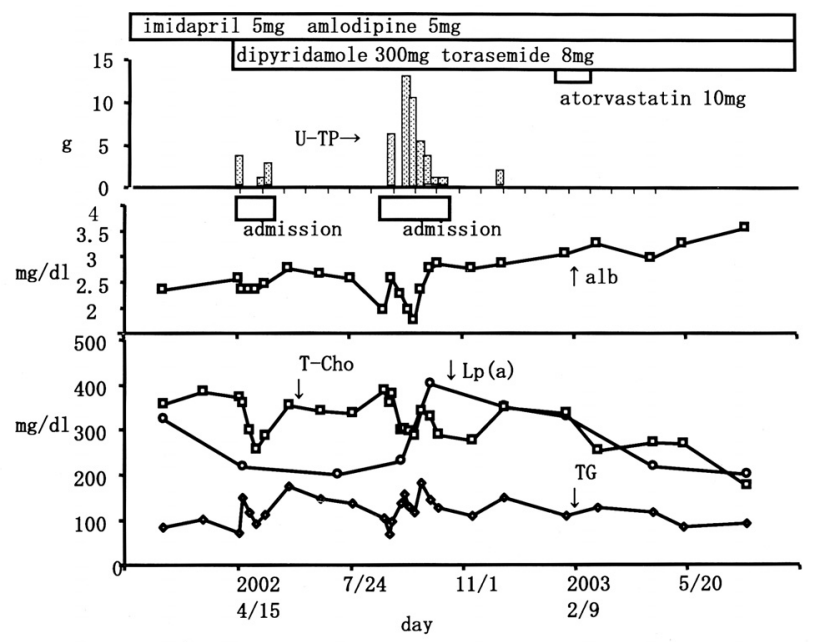

Fig. 6. Clinical course.

U-TP: urinary total protein/day, alb: plasma albumin, T-Cho: total cholesterol, TG: triglyceride

\section{Results and Discussion}

We encountered a patient with hyperLp(a)emia complicated by advanced atherosclerosis, detected when nephrotic syndrome developed during the clinical course. We found that changes in Lp(a) levels closely paralleled the severity of nephrotic syndrome. However, after remission of the nephrotic syndrome, Lp(a) levels remained high. Therefore, we estimated that marked hyper $\mathrm{Lp}(\mathrm{a})$ emia may have played a role in the progression of severe atherosclerosis in this case.

Plasma concentrations of TC and TG are frequently elevated in patients with nephrotic syndrome. This type of hyperlipoproteinemia is mainly caused by an increase in atherogenic lipoproteins, consisting of apolipoprotein B100 as a major apoprotein. (7). Concerning changes in $\mathrm{Lp}(\mathrm{a})$ levels, there have been some reports showing the elevation of plasma Lp(a) levels in nephrotic syndrome (8-11). Lp(a) levels are much less affected by age, gender, weight and diet than other classes of lipoproteins. Lp(a) plasma levels are under tight genetic control, independent of low-density lipoprotein plasma concentrations, and refractory to dietary or drug treatment. Utermann et al. (6) showed an inverse correlation between the size of apo(a) isoforms, that is, the number of repeated kringle 4 domains, and the plasma Lp(a) concentration. In the present study, the patient had a phenotype of $F, S 3$, including the small type of Lp(a). Although the size of the polymorphism does not explain all of the interindividual variations in $L p(a)$ levels (12), the small apo(a) particles may have been a factor in the elevation of $L p(a)$ levels in the present case.

Concerning the mechanism involved in increasing $\mathrm{Lp}(\mathrm{a})$ in nephrotic syndrome, De Sain-Van Der Velden et al. (13) used a stable isotope, ${ }^{13} \mathrm{C}$ valine, to demonstrate that the absolute synthetic rate of $L p(a)$ was correlated with plasma Lp(a) concentration, suggesting that increased synthesis causes elevated plasma $L p(a)$ concentrations in nephrotic syndrome. In the present study, we found that $L p(a)$ levels were relatively closely correlated with the levels of plasma TC and albumin. It has been proposed that hypoalbuminemia and reduced oncotic pressure stimulate hepatic synthesis of albumin and other liver-derived proteins, such as apoproteins (14). However, the precise mechanism driving the increased synthesis of Lp(a) has not yet been clarified.

Elevated plasma concentrations of $L p(a)$ are recognized as a risk factor for the development of atherosclerotic diseases such as CAD, peripheral vascular disease and 
stroke (15). However, clinical profiles of patients with very high levels of $L p(a)$ have not been fully clarified, although there have been a few reports associated with juvenile CAD $(16,17)$. Thiery et al. reported a case of nephrotic syndrome with hyperLP(a)emia, which also demonstrated severe CAD (8). Although the present case had some risk factors such as smoking and hypertension, high levels of $L p(a)$ may have played a role in the progression of atherosclerosis, since the combination of high Lp(a) plasma concentrations with other cardiovascular risk factors strongly increases the risk for atherosclerotic disease (18-21).

Whether high $L p(a)$ itself plays a direct role in the development of nephrotic syndrome is not clear. LDL is reported to have a worsening effect on nephrotic syndrome (22). Hyperlipoproteinemia is occasionally associated with severe glomerular injury, caused by abnormal accumulation of lipids in glomeruli, which occurs in conditions such as lipoprotein glomerulopathy (23). Since renal biopsy was not performed in the present case, the pathological relationship between the high levels of $L p(a)$ and the development of nephrotic syndrome is not clear.

Finally, extremely high plasma Lp(a) levels may play an important role in the progression of atherosclerosis. Further studies of cases showing marked hyperlipoprotein(a) emia will provide more information, further clarifying the pathological role of $L p(a)$ in atherogenesis.

\section{References}

(1) McLean JW, Tomlinson JE, Kuang WJ, Eaton DL, Chen EY, Fless GM, Scanu AM, and Lawn RM: cDNA sequence of human apolipoprotein(a) is homologous to plasminogen. Nature, 330: 132-137, 1987

( 2 ) Berg K, Dahlen G, and Frick MH: Lp(a) lipoprotein and pre-beta1-lipoprotein in patients with coronary heart disease. Clin Genet, 6: 230-235, 1974

( 3 ) Murai A, Miyahara T, Fujimoto N, Matsuda M, and Kameyama M: $L p(a)$ lipoprotein as a risk factor for coronary heart disease and cerebral infarction. Atherosclerosis, 59: 199-204, 1986

( 4 ) Abe A, Yoshimura Y, Sekine T, Maeda S, Yamashita $S$, and Noma A: Fully mechanized latex immunoassay for serum lipoprotein (a). Clinica Chim Acta, 225: 105-113, 1994

( 5 ) Abe A, Noma A, and Itakura H: Lipoprotein(a) phenotyping using a computerized micro scale and phenotype frequencies in a healthy Japanese population. Clin Chim Acta, 219: 149-157, 1993

(6) Utermann G, Menzel HJ, Kraft HG, Duba HC, Kemmler HG, and Seitz C: Lp(a) glycoprotein phenotypes. Inheritance and relation to $L p(a)$-lipoprotein concentrations in plasma. J Clin Invest, 80: 458465, 1987
( 7 ) Keane WF and Kasiske BL: Hyperlipidemia in the nephrotic syndrome. N Engl J Med, 323: 603-604, 1990

( 8 ) Thiery J, Ivandic B, Bahlmann G, Walli AK, and Seidel D: Hyperlipoprotein(a)aemia in nephrotic syndrome. Eur J Clin Invest, 26: 316-321, 1996

( 9 ) De Sain-Van Der Velden MG, Reijngoud DJ, Kaysen GA, Gadellaa MM, Voorbij H, Stellaard F, Koomans $\mathrm{HA}$, and Rabelink TJ: Evidence for increased synthesis of lipoprotein(a) in the nephrotic syndrome. J Am Soc Nephrol, 9: 1474-1481, 1998.

(10) Joven J, Simo JM, Vilella E, Camps J, Espinel E, and Villabona C: Accumulation of atherogenic remnants and lipoprotein(a) in the nephrotic syndrome: relation to remission of proteinuria. Clin Chem, 41: 908-913, 1995

(11) Faucher C, Doucet C, Baumelou A, Chapman J, Jacobs $C$, and Thillet J: Elevated lipoprotein (a) levels in primary nephrotic syndrome. Am J Kidney Dis, 22: 808-813, 1993

(12) Utermann G: Genetic architecture and evolution of the lipoprotein(a) trait. Curr Opin Lipidol, 10: 133141,1999

(13) De Sain-Van Der Velden MG, Reijngoud DJ, Kaysen GA, Gadellaa MM, Voorbij H, Stellaard F, Koomans $\mathrm{HA}$, and Rabelink TJ: Evidence for increased synthesis of lipoprotein(a) in the nephrotic syndrome. J Am Soc Nephrol, 9: 1474-1481, 1998

(14) Kaysen GA: Hyperlipidemia of the nephrotic syndrome. Kidney Int, 39: S8-S15, 1991

(15) Dahlen GH, Guyton JR, Attar M, Farmer JA, Kautz JA, Gotto and AM Jr: Association of levels of lipoprotein Lp(a), plasma lipids, and other lipoproteins with coronary artery disease documented by angiography. Circulation, 74: 758-765, 1986

(16) Bambauer R, Schiel R, Keller HE, Klinkmann J, and Latza R: Low-density lipoprotein apheresis in the treatment of two patients with coronary heart disease and extremely elevated lipoprotein (a) levels. Artif Organs, 20: 340-243, 1996

(17) Bambauer R, Schiel R, Latzo R, and Klinkmann J: LDL-apheresis in treatment of two patients with heterozygous familial hypercholesterolemia and extremely elevated lipoprotein (a) levels. Transfus Sci, 16: 375-381, 1995

(18) Kostner GM, Avogaro P, Cazzolato G, Marth E, Bittolo-Bon G, and Qunici GB: Lipoprotein Lp(a) and the risk for myocardial infarction. Atherosclerosis, 38: 51-61, 1981

(19) Armstrong VW, Cremer P, Eberle E, Manke A, Schulze F, Wieland H, Kreuzer H, and Seidel D: The association between serum Lp(a) concentrations and angiographically assessed coronary atherosclerosis. Dependence on serum LDL levels. Atherosclerosis, 62: 249-257,1986. 
(20) Dahlen GH, Weinehall L, Stenlund H, Jansson JH, Hallmans G, Huhtasaari F, and Wall S: Lipoprotein(a) and cholesterol levels act synergistically and apolipoprotein A-I is protective for the incidence of primary acute myocardial infarction in middle-aged males. An incident case-control study from Sweden. J Intern Med, 244(5): 425-430, 1998

(21) Frick MH, Dahlen G, Berg K, Valle M, and Hekali P: Serum lipids in angiographically assessed coronary atherosclerosis. Chest, 73: 62-65, 1978

(22) Muso E, Mune M, Fujii Y, Imai E, Ueda N, Hatta K,
Imada A, Takemura T, Miki, S, Kuwahara T, Takamitsu $Y$, Tsubakihara $Y$, and the Kansai FGS LDL Apheresis Treatment (K-FLAT) Study Group: Significantly rapid relief from steroid-resistant nephrotic syndrome by LDL apheresis compared with steroid monotherapy. Nephron, 89: 408-415, 2001

(23) Saito T, Sato H, Kudo K, Oikawa S, Shibata T, Hara Y, Yoshinaga K, and Sakaguchi H: Lipoprotein glomerulopathy: glomerular lipoprotein thrombi in a patient with hyperlipoproteinemia. Am J Kidney Dis, 13: 148-153, 1989. 